Article

\title{
Comparative and Phylogenetic Analyses of Ginger (Zingiber officinale) in the Family Zingiberaceae Based on the Complete Chloroplast Genome
}

\author{
Yingxian Cui ${ }^{1,3}$, Liping Nie ${ }^{1,3}$, Wei Sun ${ }^{2}{ }^{(0)}$, Zhichao Xu ${ }^{1,3}$, Yu Wang ${ }^{1,3}$, Jing Yu ${ }^{1}$, \\ Jingyuan Song ${ }^{1,3}$ and Hui Yao ${ }^{1,3, *(D)}$ \\ 1 Key Lab of Chinese Medicine Resources Conservation, State Administration of Traditional Chinese Medicine \\ of the People's Republic of China, Institute of Medicinal Plant Development, Chinese Academy of Medical \\ Sciences and Peking Union Medical College, Beijing 100193, China \\ 2 Institute of Chinese Materia Medica, China Academy of Chinese Medical Sciences, Beijing 100700, China \\ 3 Engineering Research Center of Chinese Medicine Resources, Ministry of Education, Beijing 100193, China \\ * Correspondence: scauyaoh@sina.com; Tel.: +86-10-57833194
}

Received: 18 July 2019; Accepted: 11 August 2019; Published: 12 August 2019

check for updates

\begin{abstract}
Zingiber officinale, commonly known as ginger, is an important plant of the family Zingiberaceae and is widely used as an herbal medicine and condiment. The lack of chloroplast genomic information hinders molecular research and phylogenetic analysis on ginger. We introduced the complete chloroplast genome of $Z$. officinale and identified its phylogenetic position in Zingiberaceae. The chloroplast genome of $Z$. officinale is $162,621 \mathrm{bp}$ with a four-part circular structure and $36.1 \%$ GC content. All 113 unique genes were annotated. A total of 78 simple sequence repeats (SSRs) and 42 long repeat sequences, which are potential areas for species authentication, were found. Comparative analysis revealed some highly variable regions, including $r p s 16$-trnQ-UUG, atpH-atpI, trnT-UGU-trnL-UAA, ycf1, and $p s a C$-ndhE. Moreover, the small single-copy (SSC) region was the most variable region in all four shared regions, indicating that it may be undergoing rapid nucleotide substitution in the family Zingiberaceae. Phylogenetic analysis based on all available chloroplasts of Zingiberales in the National Center for Biotechnology Information indicated that Zingiber is a sister branch to Kaempferia species. The availability of the Z. officinale chloroplast genome provided invaluable data for species-level authentication and phylogenetic analysis and can thus benefit further investigations on species in the family Zingiberaceae.
\end{abstract}

Keywords: Zingiber officinale; Zingiberaceae; complete chloroplast genome; comparative analysis; phylogenetic analysis

\section{Introduction}

Zingiber officinale, commonly known as ginger, is a common plant of the family Zingiberaceae that is universally cultivated in the Central, Southeastern, and Southwestern provinces of China, as well as in tropical Asia [1]. Its rhizome is a common plant material with medicinal and condiment properties. In China, its fresh and dried forms with different therapeutic effects are recorded in the Chinese Pharmacopoeia [2]. Currently, ginger has gained attention as researchers has demonstrated that its compounds can be used to treat cardiovascular disease [3,4] and have antioxidant $[5,6]$, anti-inflammatory [4,7], analgesic, immunomodulatory, antitumorigenic [7,8], and anti-emetic [9] properties with few side effects. Increasing attention has focused on the genetic engineering and molecular research of ginger in recent years as a result of its medicinal effects and edibility [10]. However, to the best of our knowledge, the chloroplast genome of ginger is still unclear. Only the chloroplast genome sequences of six species in the whole family Zingiberaceae were recorded in the 
National Center for Biotechnology Information (NCBI). Hence, more information should be obtained about the chloroplast genome resources of the Zingiberaceae species.

The complete chloroplast genome of angiosperms usually consists of four parts: A large single-copy (LSC) region, small single-copy (SSC) region, and two similar inverted repeat (IR) regions [11]. Unlike the nuclear genome, the chloroplast genome of most angiosperms has the characteristics of maternal inheritance, highly conserved gene content and order, and lower recombination rate, making it an ideal material for species authentication and phylogenetic studies [12-15]. Highly variable regions on the chloroplast genomes have been revealed and successfully used for species authentication, such as in Rhubarb botanical [16] and Ipomoea species [17]. Moreover, some studies have suggested that complete chloroplast genomes can be used as the super-code to identify related species, which is a challenge when using traditional molecular markers, such as in the case of Ephedra species [18], grass species [19], and Dendrobium species [20]. The availability of complete chloroplast genomes has greatly contributed to resolving the evolutionary and phylogenetic relationships of angiosperms $[19,21,22]$.

Here, we introduce the complete chloroplast genomes of Z. officinale and compare the obtained sequences with reported chloroplast genomes in the family Zingiberaceae. The availability of the $Z$. officinale chloroplast genome will greatly contribute to species authentication, phylogenetic analysis, and genetic engineering study of species in the family Zingiberaceae.

\section{Results and Discussion}

\subsection{Zingiber Officinale Chloroplast Genome Features}

Approximately $8.5 \mathrm{~Gb}$ of raw data from Z. officinale with pair-end $150 \mathrm{bp}$ read lengths were obtained. The Z. officinale chloroplast genome is $162,621 \mathrm{bp}$ and contains four regions, including an LSC ( $87,486 \mathrm{bp})$ region, SSC ( $15,577 \mathrm{bp})$ region, and two identical IR ( $29,779 \mathrm{bp})$ regions, as shown in Table 1 and Figure 1. The overall GC contents of the Z. officinale chloroplast genome and protein-coding regions were $36.1 \%$ and $37.1 \%$, respectively. Both IR $(41.1 \%)$ regions possess all four rRNA genes with high GC content in the genome-higher than those of the LSC $(33.8 \%)$ and SSC $(29.7 \%)$ regions (Table 1$)$. The AT content $(71.1 \%)$ in the third codon position was remarkably higher than those in the first $(55.2 \%)$ and second codon positions $(62.2 \%)$ within the protein-coding regions of $Z$. officinale chloroplast genomes (Table 1). These observations were similar to those for other reported plants [21,23-25] and were used as identification characteristics to discern chloroplast DNA from nuclear and mitochondrial DNA.

Table 2 shows the 113 unique genes that were identified in the Z. officinale chloroplast genome, which included 79 protein-coding genes, 30 tRNA genes, and four rRNA genes. Twenty genes (eight protein-coding genes, eight tRNA genes and four rRNA genes) were duplicated in the IR regions, resulting in 133 genes located in the complete Z. officinale chloroplast genome. The LSC regions contained 60 protein-coding genes and 21 tRNA genes, whereas the SSC regions contained 11 protein-coding genes and one tRNA gene. A total of 10 protein-coding genes and seven tRNA genes contained one intron, whereas three genes ( $r p s 12, \operatorname{trnK}-U U U, y c f 3)$ contained two introns, as shown in Table S1. Among them, the trnK-UUU gene has the largest intron, with a size of $>2500 \mathrm{bp}$, as it contains the $m a t K$ gene.

Table 1. Base composition of the Z. officinale chloroplast genome.

\begin{tabular}{ccccccc}
\hline Region & Positions & T(U) (\%) & C (\%) & A (\%) & G (\%) & Length (bp) \\
\hline Total & & 32.2 & 18.4 & 31.7 & 17.7 & 162,621 \\
IRa & 30.1 & 21.3 & 28.8 & 19.8 & 29,779 \\
IRb & & 28.8 & 19.8 & 30.1 & 21.3 & 29,779 \\
LSC & 33.7 & 17.4 & 32.4 & 16.5 & 87,486 \\
SSC & & 34.3 & 15.8 & 36.0 & 13.9 & 15,577 \\
CDS & 31.7 & 17.2 & 31.2 & 19.9 & 79,167 \\
& 1st position & 24 & 18.3 & 31.3 & 26.5 & 26,389 \\
& 2nd position & 33 & 20.1 & 29.7 & 17.7 & 26,389 \\
& 3rd position & 39 & 13.2 & 32.6 & 15.6 & 26,389 \\
\hline
\end{tabular}




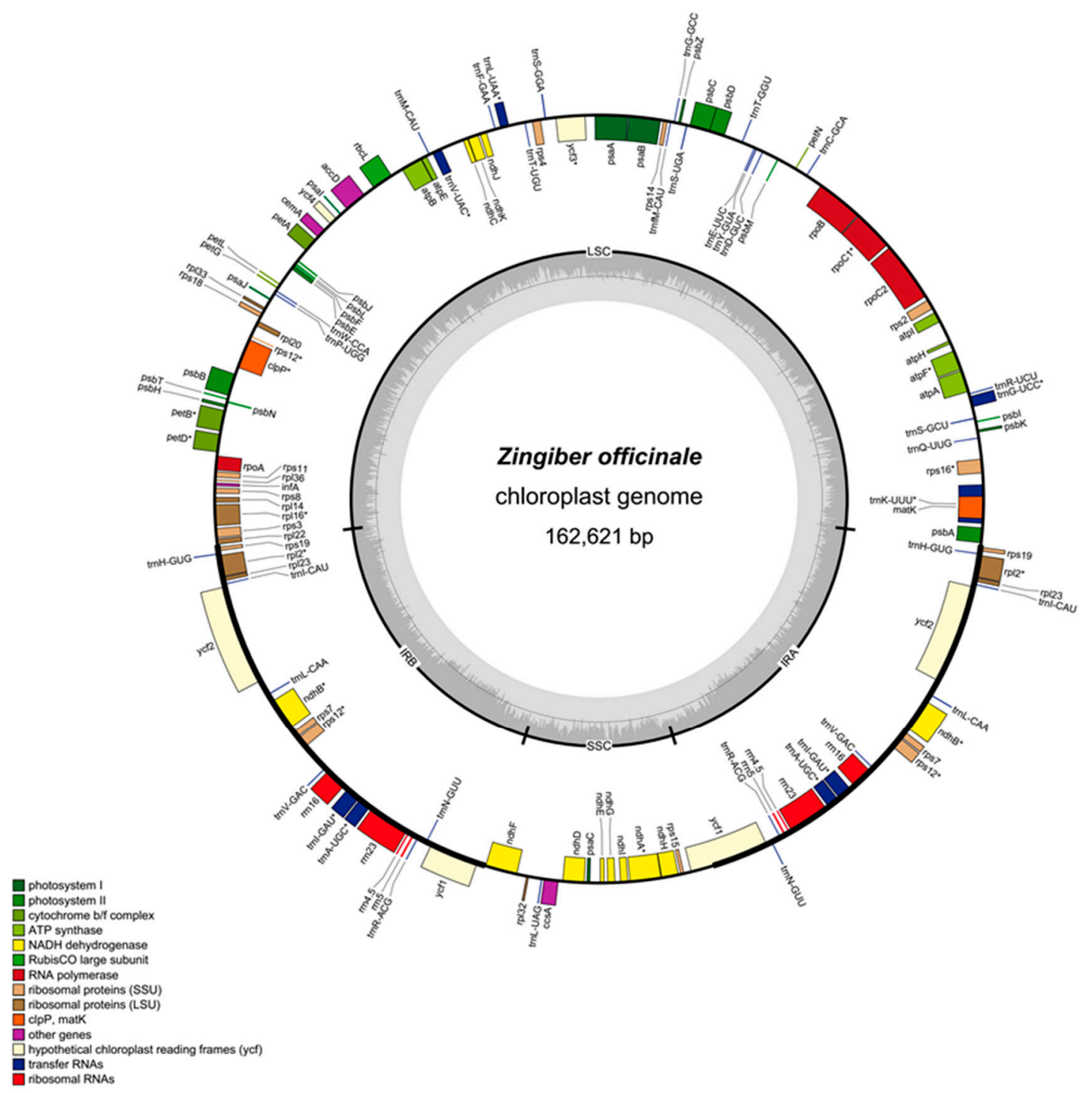

Figure 1. Gene map of the Zingiber officinale complete chloroplast genome. Genes that are inside and outside the circle are transcribed clockwise and counterclockwise, respectively. The darker gray area in the inner circle corresponds to GC content, whereas the lighter gray area corresponds to AT content.

Table 2. Gene content of the Z. officinale chloroplast genome.

\begin{tabular}{|c|c|c|}
\hline Group of Genes & Gene Names & Amount \\
\hline Photosystem I & $p s a A, p s a B, p s a C, p s a I, p s a J$ & 5 \\
\hline Photosystem II & $p s b A, p s b B, p s b C, p s b D, p s b E, p s b F, p s b H, p s b I, p s b J, p s b K, p s b L, p s b M, p s b N, p s b T, p s b Z$ & 15 \\
\hline Cytochrome b/f complex & $\operatorname{pet} A, \operatorname{pet} B^{*}, \operatorname{pet} D^{*}, \operatorname{pet} G, \operatorname{pet} L, \operatorname{pet} N$ & 6 \\
\hline NADH dehydrogenase & $n d h A^{*}, n d h B^{*}(\times 2), n d h C, n d h D, n d h E, n d h F, n d h G, n d h H, n d h I, n d h J, n d h K$ & 12 \\
\hline RubisCO large subunit & $r b c L$ & 1 \\
\hline RNA polymerase & rpoA, rpoB, rpoC1*, rpoC2 & 4 \\
\hline Proteins of unknown function & $y c f 1(\times 2), y c f 2(\times 2), y c f 3^{* *}, y c f 4$ & 6 \\
\hline Transfer RNAs & 38 tRNAs $(8$ in the IRs $(\times 2)) * *$ & 38 \\
\hline Ribosomal RNAs & $r r n 4.5(\times 2), r r n 5(\times 2), r r n 16(\times 2), r r n 23(\times 2)$ & 8 \\
\hline Other genes & $a c c D, \operatorname{clp} P^{*}, \operatorname{mat} K, \operatorname{ccs} A, \operatorname{cem} A, \inf A$ & 6 \\
\hline
\end{tabular}

$(\times 2)$ indicates that the number of the repeating unit is $2 ;{ }^{*}$ indicates introns of genes. 


\subsection{Codon Usage Analysis}

The chloroplast genome of Z. officinale was analyzed for its codon usage frequency based on the sequence of protein-coding genes and on relative synonymous codon usage (RSCU). RSCU refers to the relative probability of a codon encoding a corresponding amino acid synonymous codon, which removes the effect of amino acid composition on codon usage. The RSCU value of this codon is equal to one if codon use is not preferred, whereas a value greater than one means a relatively large number of codons, and vice versa. A total of 63 codons encoded 20 amino acids. All annotated genes of Z. officinale were encoded by 26,389 codons, as shown in Figure 2 and Table S2. Similarly shown in this figure and table is that among the amino acids, leucine, with 2732 (10.4\%) codons, occurred most frequently, whereas cysteine $303(1.1 \%)$ was observed to have the least occurrence. Most of the amino acid codons have preferences except for methionine (AUG) and tryptophan (UGG), whose RSCU values are equal to one. A majority of the preferred synonymous codons (RSCU $>1$ ) possess A- or U-ending codons, except for $t r n L-C A A$, which is encoded by UUG (Figure 2 and Table S2). Codons ending with $\mathrm{A}$ and/or $\mathrm{U}$ accounted for $71.2 \%$, resulting in the bias for A/T bases. Similar patterns were observed in other reported chloroplast genomes, such as in Ulmus [26], Papaver [27], Lycium [28], and Taxillus [11] species.

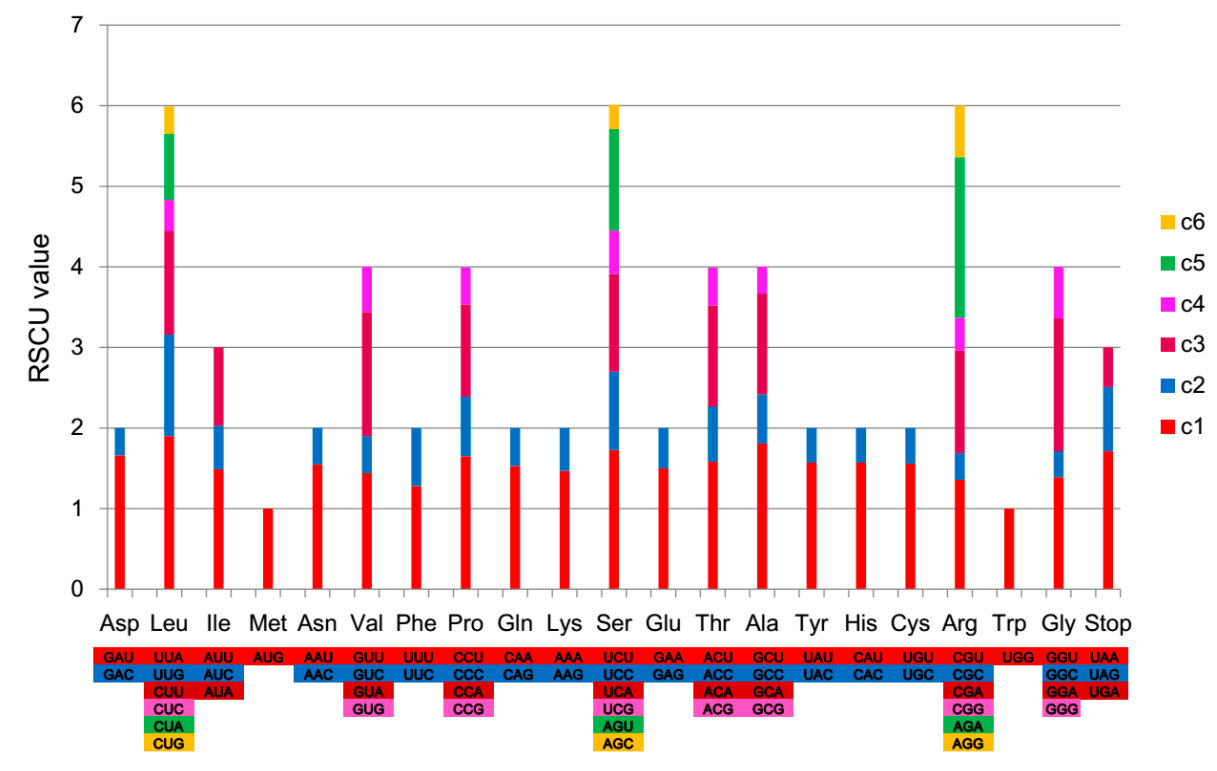

Figure 2. Codon content of all protein-coding genes in the Z. officinale chloroplast genome.

\subsection{Repeat Structure Analysis}

Simple sequence repeats (SSRs), also called microsatellites, are present throughout genomes and consist of tandem repeats of one to six nucleotides [29]. Most SSRs have high levels of polymorphism and are widely used for species authentication, phylogenetic analysis, and population genetics [17,30-32]. In total, 78 SSRs were identified in the Z. officinale chloroplast genome, including 27 mononucleotide, 23 dinucleotide, five trinucleotide, 21 tetranucleotide, and two pentanucleotide repeats (Table 3 and Table S3). Among them, 54, 14, and five SSRs were distributed in the LSC, SSC, and IR regions, respectively. A/T mononucleotide repeats (34.6\%) were observed to be most common, followed by AT/TA dinucleotide repeats (26.9\%), as shown in Table 3. Furthermore, A and T were the most frequent bases in all SSR types, which resulted in the bias in base in the Z. officinale chloroplast genome. 
Table 3. Types and amounts of simple sequence repeats (SSRs) in the Z. officinale chloroplast genome.

\begin{tabular}{cccc}
\hline SSR Type & Repeat Unit & Amount & Ratio (\%) \\
\hline Mono & A/T & 27 & 100.0 \\
& AG/CT & 2 & 8.7 \\
Di & AT/TA & 21 & 91.3 \\
& AAG/CTT & 1 & 20.0 \\
& AAT/ATT & 4 & 80.0 \\
& AAAC/GTTT & 1 & 4.8 \\
\multirow{5}{*}{ Tetra } & AAAG/CTTT & 4 & 19.0 \\
& AAAT/ATTT & 13 & 61.9 \\
& AACT/AGTT & 1 & 4.8 \\
& AATG/ATTC & 2 & 9.5 \\
Penta & AAAAT/ATTTT & 1 & 50.0 \\
& AATAG/ATTCT & 1 & 50.0 \\
\hline
\end{tabular}

Some $>30$ bp structures are known as long repeat sequences, which might be helpful to phylogenetic analysis and which increase chloroplast genome rearrangement [33]. Figure 3 shows 42 long repeats in the Z. officinale chloroplast genome. Of the identified repeats, 32 have sizes between 30 and $39 \mathrm{bp}$, including 13 forward, 13 palindromic, five reverse, and one complement repeat. Moreover, five forward and four palindromic repeats are $40-49 \mathrm{bp}$, whereas one forward repeat is more than $70 \mathrm{bp}$.

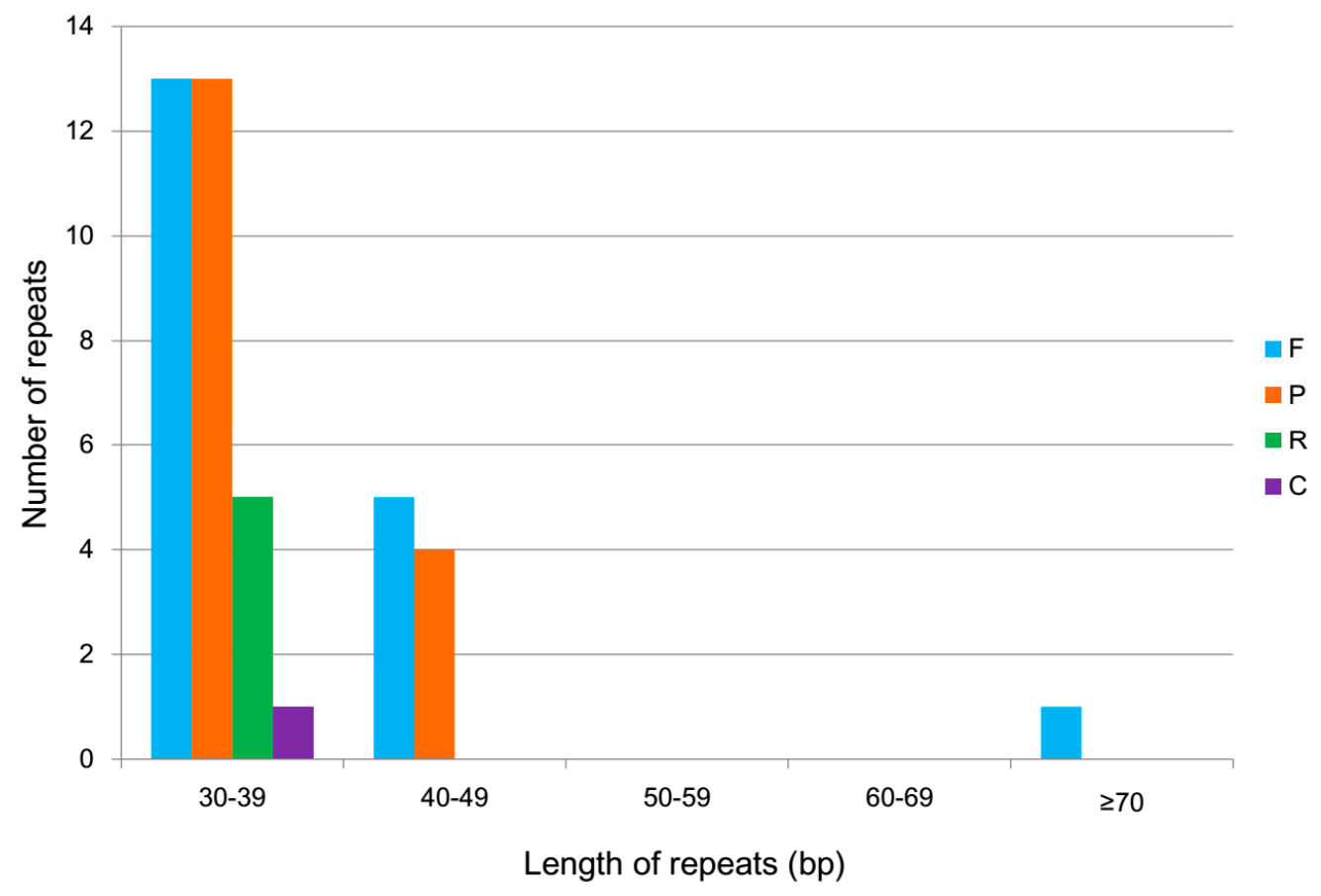

Figure 3. Repeat sequences in the Z. officinale chloroplast genome. F, forward; P, palindrome; R, reverse; and $\mathrm{C}$, complement.

\subsection{Comparative Analysis}

The Z. officinale chloroplast genome was compared to those of species in the family Zingiberaceae (Z. spectabile, Kaempferia elegans, K. galangal, Curcuma longa, C. flaviflora, C. roscoeana), with the annotated $Z$. officinale sequence as a reference (Figure 4). Among these species, Z. officinale had the third longest chloroplast genome, following K. elegans and K. galangal. It is larger than that of Z. spectabile by $6731 \mathrm{bp}$. The result showed that the non-coding regions appeared to be more variable than the coding regions, the two IR regions were less divergent than that LSC and SSC regions, four rRNA genes were the most conserved regions, and the intergenic spacers were the most divergent regions. The highly 
divergent regions among the seven chloroplast genomes occurred in the intergenic spacers, including rps16-trnQ-UUG, atpH-atpI, trnT-UGU-trnL-UAA, ycf1, and $p s a C-n d h E$, regions of which had been also observed in other plant chloroplast genomes in Zingiberaceae [21,24,28]. Moreover, $y c f 1, y c f 2$, and $n d h F$ were the most variable coding regions in these species.

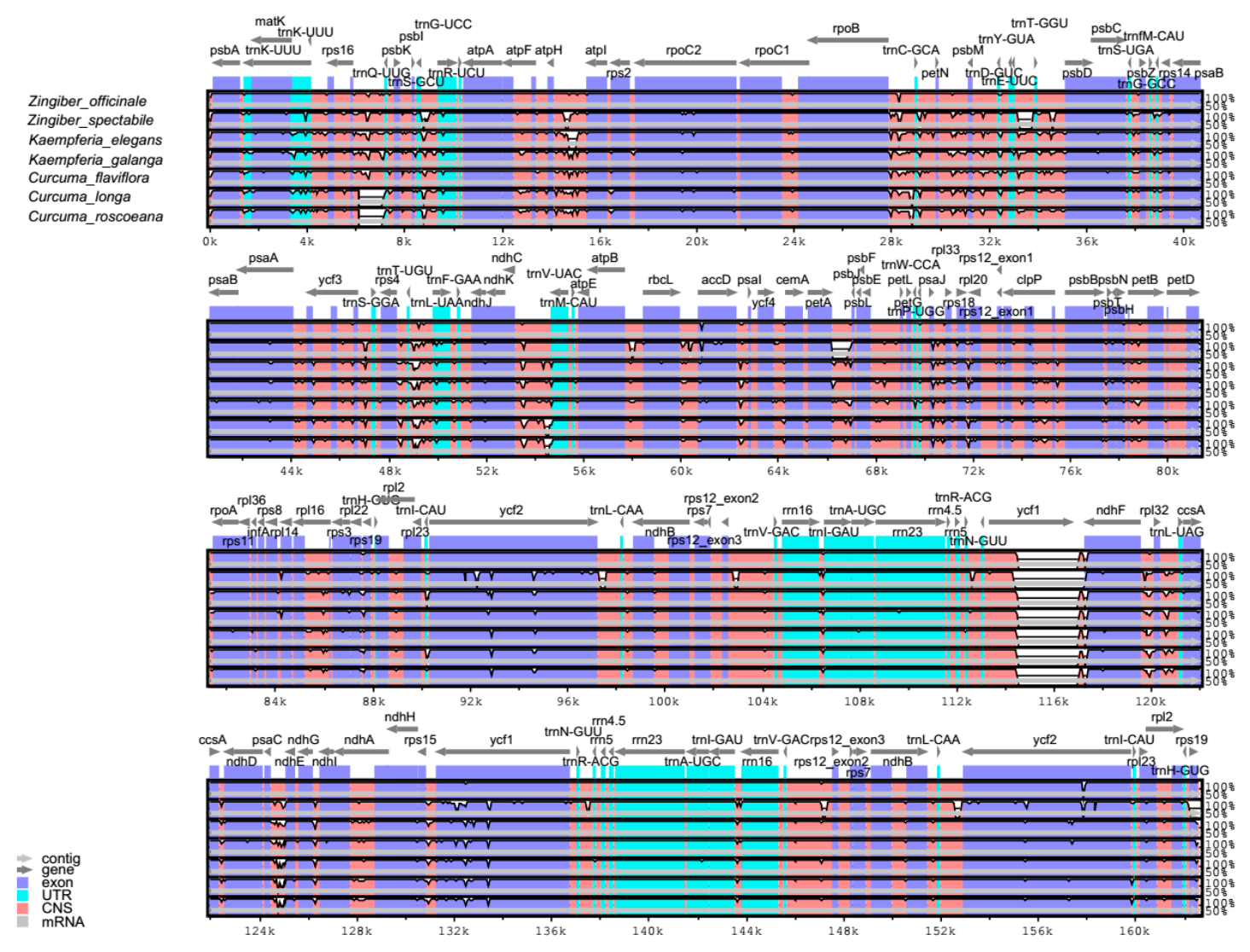

Figure 4. Comparison of the complete chloroplast genomes of seven species in the family Zingiberaceae using mVISTA, with Z. officinale as a reference. Blue block, conserved genes; sky-blue block, transfer RNA (tRNA) and ribosomal RNA (rRNA); and red block, conserved noncoding sequences (CNS).

White represents regions with sequence variation among the seven species.

Moreover, 10 species in Zingiberaceae (Z. officinale, Z. spectabile, K. elegans, K. galangal, C. longa, C. flaviflora, C. roscoeana, Amomum compactum, A. krervanh, and Alpinia oxyphylla) were observed to have highly variable regions in their chloroplast genomes by sliding window analysis using DnaSP software (Figure 5). The results showed that the average value of nucleotide variability (PI) of all the 10 species was 0.0187 , and that of the four relatively related species (Z. officinale, Z. spectabile, $K$. elegans, and K. galang) was 0.0075 . The IR regions were observed to have lower PI value than the other regions. Moreover, the SSC regions were the most variable among all four regions of the complete chloroplast genomes in Zingiberaceae. Furthermore, some regions with high PI values $(>0.115$ or $>0.025$ ) were observed in the SSC region. The results indicated that the SSC region may be undergoing rapid nucleotide substitution in species of family Zingiberaceae and may contain more important variable information for species authentication and phylogenetic analysis. 


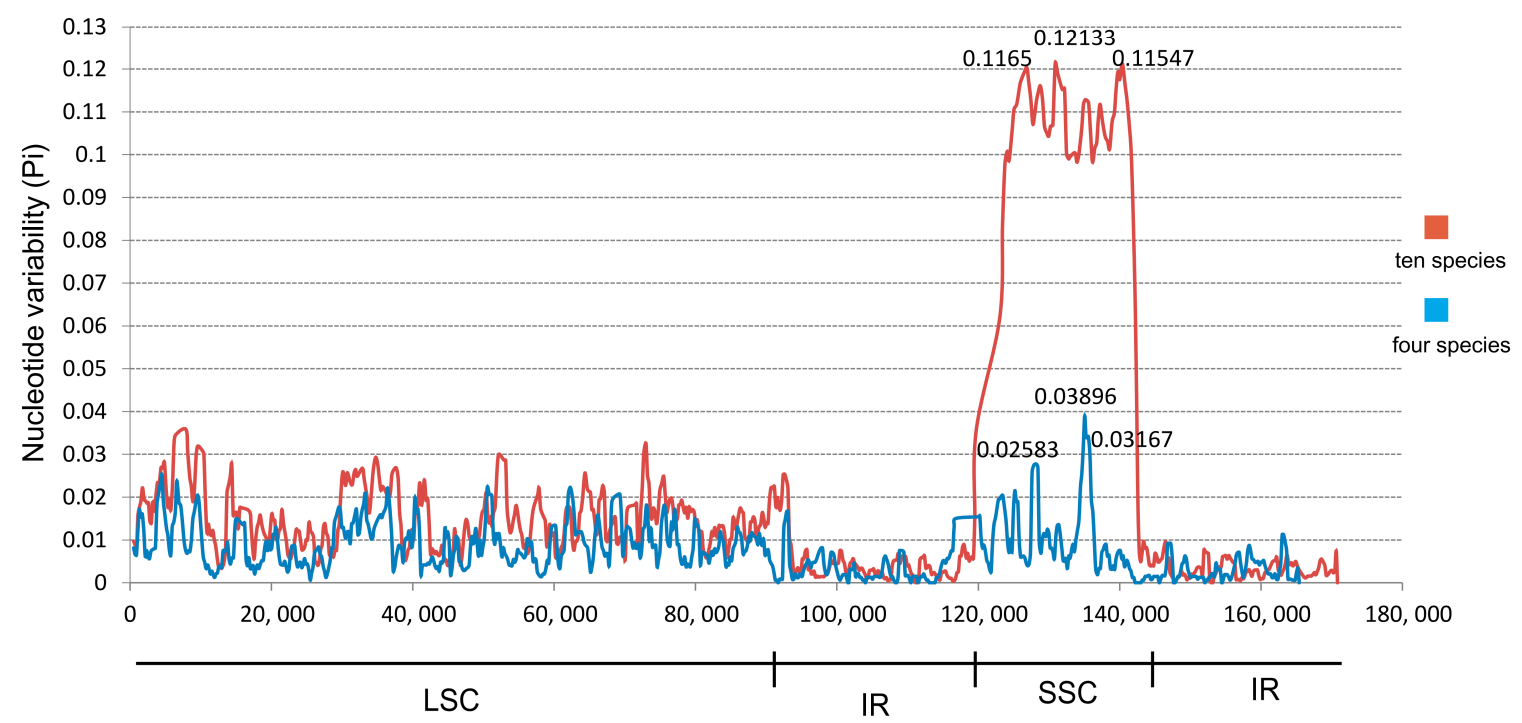

Figure 5. Sliding window analysis of the Z. officinale chloroplast genome. The red line indicates the comparison of 10 species in the family Zingiberaceae (Z. officinale, Z. spectabile, K. elegans, K. galangal, C. longa, C. flaviflora, C. roscoeana, Amomum compactum, A. krervanh, and Alpinia oxyphylla); the blue line indicates the comparison of four species in the family Zingiberaceae (Z. officinale, Z. spectabile, K. elegans, and K. galang). Window length: 800 bp; step size 200 bp. $x$ axis: Position of the midpoint of a window. $y$ axis: nucleotide diversity of each window.

\subsection{Phylogenetic Analysis}

In recent years, more chloroplast genome sequences have been identified using rapid and high-throughput sequencing [34,35]. The continuously expanding chloroplast genome database provides an important basis for the determination of the evolutionary and phylogenetic relationships of plants. Here, to reveal the phylogenetic position of Z. officinale in Zingiberales, 19 plasmid genomes were downloaded from GenBank, which contained all the available chloroplast genomes in Zingiberales. From phylogenetic analysis (Figure 6), Zingiberales species were basically divided into two branches (bootstrap value $=100 \%$ ): Clade A and Clade B. On the one hand, Clade A consisted of species in the Zingiberaceae and Strelitziaceae families, indicating that these families had a closer relationship in Zingiberales. Z. officinale is a sister species to Z. spectabile with bootstrap values of $100 \%$ from the same clade. The result also provided evidence that Zingiber species are a sister-branch to the Kaempferia species. Species in Zingiber, Kaempferia, and Curcuma being clustered into a phylogenetic group demonstrated a close relationship among them. Two species in Amomum were clustered into a branch with Alpinia oxyphylla. On the other hand, Clade B contained the Musaceae and Heliconiaceae families and all species in Musa clustered into a monophyletic clade, which had a close relationship with Musella lasiocarpa, followed by Heliconia collinsiana. The results also indicated that the chloroplast genome database is helpful to phylogenetic analysis of species in the family Zingiberaceae. It will also be a useful resource for molecular phylogeny studies within the order Zingiberales. 

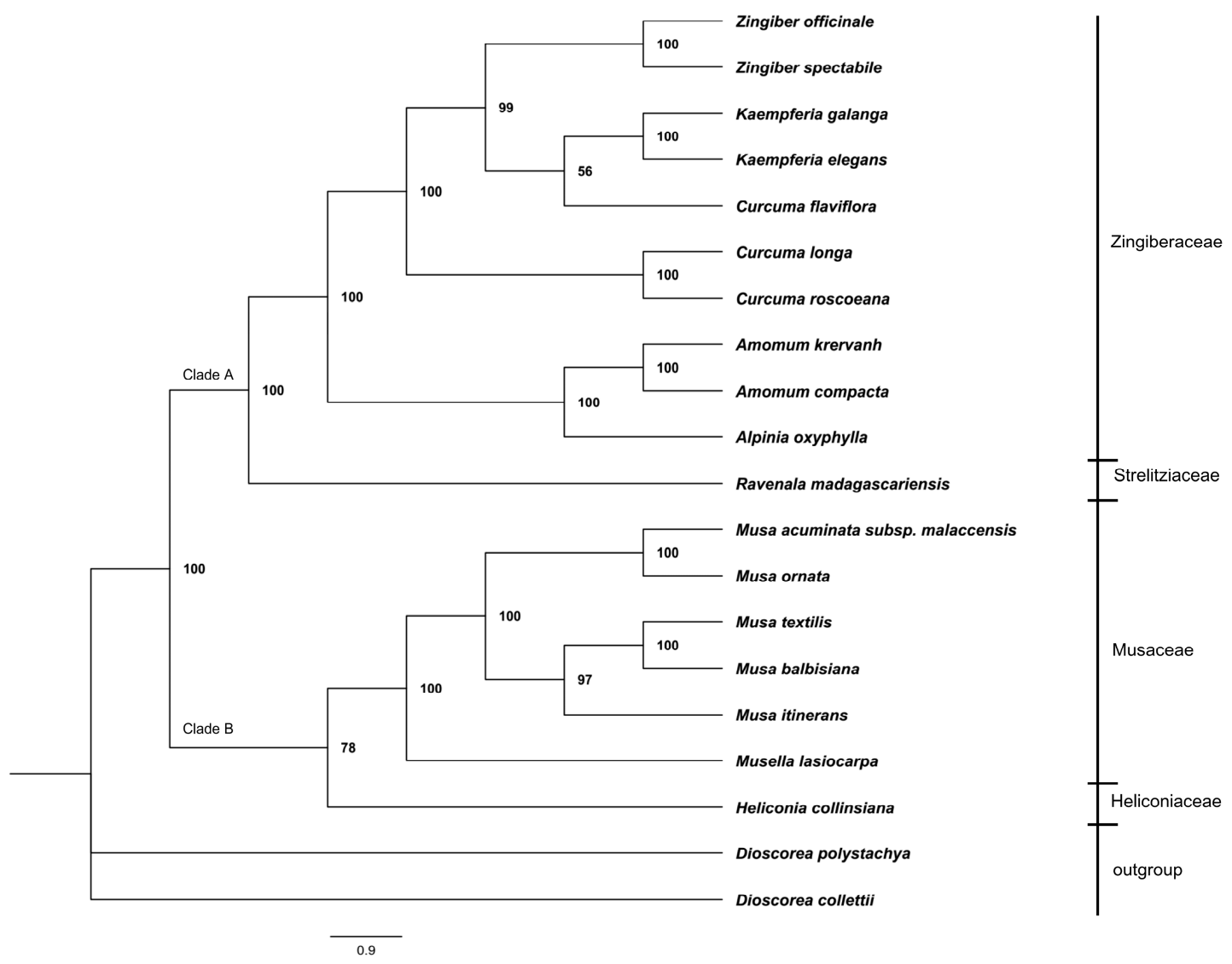

Figure 6. Likelihood (ML) phylogenetic tree of 19 species in order Zingiberales based on the concatenated sequences of 75 shared protein-coding genes of chloroplast genomes. Numbers at nodes are values for bootstrap support. Dioscorea polystachya and D. collettii were used as outgroups.

\section{Materials and Methods}

\subsection{Plant and DNA Sources}

Fresh Z. officinale leaves were collected from Laiwu City, Shandong Province, China, and were identified by Prof. Yulin Lin from the Institute of Medicinal Plant Development (IMPLAD), Chinese Academy of Medical Sciences (CAMS). The voucher specimens were deposited in the herbarium of IMPLAD. Total genomic DNA was extracted from the clean sample leaves using a DNeasy Plant Mini Kit following standard protocol (Qiagen Co., Hilden, Germany) and the DNA concentration and quality were respectively assessed through Nanodrop 2000C spectrophotometry and electrophoresis in $1 \%(w / v)$ agarose gel. Approximately $1 \mu \mathrm{g}$ genomic DNA was used for sequencing library construction. Paired-end libraries with insert sizes of $400 \mathrm{bp}$ were prepared following Illumina's standard genomic DNA library preparation procedure. Total DNA was sequenced in Illumina HiSeq X.

\subsection{Chloroplast Genome Assembly and Annotation}

Raw sequencing data were generated by Illumina and the low-quality reads were trimmed using Trimmomatic v0.36 software [36] with default parameters. Clean data obtained by the above quality control processes were used to assemble the chloroplast genome. All available chloroplast genomes of plants recorded in NCBI were downloaded to construct a basic local alignment search tool (BLASTn) database for mapping. Then, BLASTn with the default parameter was used to map and compare obtained reads to the reference chloroplast database and screen the chloroplast-like reads. Next, these reads were assembled using ABySS 2.0 [37] with multiple-Kmer parameters, and the optimal results of the assembly were obtained. SOAPdenovo2 [38] and SSPACE [39] were used to create 
the contigs and scaffolds of the chloroplast genome, respectively. GapCloser $[38,40]$ software was subsequently applied to fill up the remaining local inner gaps and correct the single base polymorphism for the final assembly results. To check the accuracy of the assembly, four junctions between the IRs and SSC/LSC regions were verified by PCR amplification and Sanger sequencing using newly designed primers (Table S4). Then, the complete chloroplast genome was acquired.

CPGAVAS [41] and DOGMA [42] were used to annotate the complete chloroplast genome with default settings followed by manual corrections. tRNAscan-SE [43] software was applied to identify the tRNA genes and BLAST search was used to annotate boundaries of genes, introns/exons and coding regions. The online software OGDRAW v1.2 [44] was programed to draw the circular map of the complete chloroplast. Finally, the complete and correct chloroplast genome of Z. officinale was submitted to GenBank with the accession number MH161428.

\subsection{Chloroplast Genome Structure and Comparison Analysis}

The software MEGA6.0 [45] was used to calculate the GC content and investigate the distribution of codon usage with the RSCU ratio. The software MISA [46] was used to detect SSRs with the parameters set to 10 repeat units $(>10)$ for mononucleotide SSRs, five repeat units $(>5)$ for dinucleotide, four repeat units $(>4)$ for trinucleotide, and three repeat units $(>3)$ for tetranucleotide, pentanucleotide, and hexanucleotide SSRs. The size and location of repeat sequences, including forward, palindromic, reverse, and complement repeats, were identified by REPuter [47]. For all repeat types, the minimal size was $30 \mathrm{bp}$ and the two repeat copies had at least $90 \%$ similarity. The whole chloroplast genomes were aligned using the online software MAFFT [48]. Whole-genome alignment of Z. officinale and other species in Zingiberaceae downloaded from Genbank was compared by mVISTA [49] and calculated suing DnaSP [50] software to determine the nucleotide diversity with $200 \mathrm{bp}$ step size and $800 \mathrm{bp}$ window length.

\subsection{Phylogenetic Analyses}

For phylogenetic analysis, a total of 19 complete chloroplast genomes were downloaded from NCBI (Table S5), and 75 common protein-coding genes shared in these chloroplast genomes were applied to construct the ML phylogenetic tree. Firstly, all shared genes from these chloroplast genomes were extracted and aligned separately by multiple alignment using MAFFT [48] and were manually adjusted. Secondly, all aligned gene sequences were concatenated and verified. Lastly, phylogenetic trees were reconstructed based on 75 concatenated protein-coding gene sequences by ML methods with a bootstrap of 1000 repetitions. IQ-TREE [51] software was employed to find the best substitution model and construct the ML phylogenetic tree.

\section{Conclusions}

Here, we sequenced and analyzed the Z. officinale chloroplast genome. Firstly, the basic structures, gene information, and codon usage pattern were revealed. Secondly, 78 SSRs and 42 long repeat sequences were identified. Thirdly, a comparative analysis within the family Zingiberaceae was executed and some variable regions which have the potential to become DNA markers were revealed. The results showed that SSC was the most variable region and may be undergoing rapid nucleotide substitution in Zingiberacelae species. The ML tree indicated that the Zingiber species has a close relationship with species in Kaempferia and Curcuma and clearly show a phylogenetic relationship with species in the family Zingiberaceae, or even the order Zingiberacelae, through molecular methods. This study provided invaluable data for species authentication and phylogenetic analysis of plants in the family Zingiberaceae. 
Supplementary Materials: The following are available online at http://www.mdpi.com/2223-7747/8/8/283/s1, Table S1: Gene with introns in Z. officinale chloroplast genome, Table S2: Codon usage of Z. officinale chloroplast genome, Table S3: Simple sequence repeats (SSRs) in Z. officinale chloroplast genome, Table S4: Primer sequences at the boundaries between single copy and IR regions of Z. officinale, Table S5: GenBank accession numbers of 19 complete chloroplast genome sequences used for ML phylogenetic analyses.

Author Contributions: Conceptualization, Y.C.; Data curation, Y.C., L.N., J.Y. and J.S.; Formal analysis, Y.C. and H.Y.; Funding acquisition, H.Y.; Investigation, L.N.; Methodology, Y.C., L.N., Z.X. and H.Y.; Project administration, Y.C.; Resources, Y.W., J.Y. and H.Y.; Software, W.S. and Z.X.; Supervision, Y.C., Y.W., J.S. and H.Y.; Validation, W.S.; Visualization, L.N., W.S. and Z.X.; Writing—original draft, Y.C.; Writing—review \& editing, H.Y.

Funding: This research was funded by Major Scientific and Technological Special Project for "Significant New Drugs Creation" (No. 2018ZX09711001-008-007) and Chinese Academy of Medical Sciences (CAMS) Innovation Fund for Medical Sciences (CIFMS) (NO. 2016-I2M-3-016).

Conflicts of Interest: The authors declare no conflict of interest.

\section{Abbreviations}

$\begin{array}{ll}\text { LSC } & \text { large single copy } \\ \text { SSC } & \text { small single copy } \\ \text { IR } & \text { inverted repeat } \\ \text { ML } & \text { maximum likelihood } \\ \text { SSR } & \text { Simple sequence repeats } \\ \text { ATP } & \text { Adenosine triphosphate } \\ \text { NADH } & \text { Nicotinamide adenine dinucleotide }\end{array}$

\section{References}

1. Flora of China; Sci. Press: Beijing, China; Mo. Bot. Gard. Press: St. Louis, MO, USA, 2008; Volume 16, p. 141. Available online: http://www.efloras.org/flora_person_list.aspx?flora_id=2\&volume_id=2016 (accessed on 16 August 2019).

2. Commission, C.P. Pharmacopoeia of the People's Republic of China; China Medical Science Press: Beijing, China, 2015.

3. Kadnur, S.V.; Goyal, R.K. Beneficial effects of Zingiber officinale Roscoe on fructose induced hyperlipidemia and hyperinsulinemia in rats. Indian J. Exp. Biol. 2005, 43, 1161. [CrossRef] [PubMed]

4. Thomson, M.; Al-Qattan, K.K.; Al-Sawan, S.M.; Alnaqeeb, M.A.; Khan, I.; Ali, M. The use of ginger (Zingiber officinale rosc.) As a potential anti-inflammatory and antithrombotic agent. Prostag. Leukotr. Ess. 2002, 67, 475-478. [CrossRef]

5. Stoilova, I.; Krastanov, A.; Stoyanova, A.; Denev, P.; Gargova, S. Antioxidant activity of a ginger extract (Zingiber officinale). Food Chem. 2007, 102, 764-770. [CrossRef]

6. Ahmed, R.S.; Seth, V.; Banerjee, B.D. Influence of dietary ginger (Zingiber officinales Rosc) on antioxidant defense system in rat: Comparison with ascorbic acid. Indian J. Exp. Biol. 2000, 38, 604-606. [PubMed]

7. Habib, S.H.M.; Makpol, S.; Hamid, N.A.A.; Das, S.; Wan, Z.W.N.; Yusof, Y.A.M. Ginger extract (Zingiber officinale) has anti-cancer and anti-inflammatory effects on ethionine-induced hepatoma rats. Clinics 2008, 63, 807-813. [CrossRef] [PubMed]

8. Pereira, M.M.; Haniadka, R.; Chacko, P.P.; Palatty, P.L.; Baliga, M.S. Zingiber officinale Roscoe (ginger) as an adjuvant in cancer treatment: A review. J. Buon 2011, 16, 414-424. [CrossRef]

9. Phillips, S.; Ruggier, R.; Hutchinson, S.E. Zingiber officinale (ginger)-an antiemetic for day case surgery. Anaesthesia 2010, 48, 715-717. [CrossRef] [PubMed]

10. Huang, J.L.; Cheng, L.L.; Zhang, Z.X. Molecular cloning and characterization of violaxanthin de-epoxidase (VDE) in Zingiber officinale. Plant Sci. 2007, 172, 228-235. [CrossRef]

11. Li, Y.; Zhou, J.; Chen, X.; Cui, Y.; Xu, Z.; Li, Y.; Song, J.; Duan, B.; Yao, H. Gene losses and partial deletion of small single-copy regions of the chloroplast genomes of two hemiparasitic Tax i llus species. Sci. Rep. 2017, 7, 12834. [CrossRef]

12. Wolfe, K.H.; Li, W.H.; Sharp, P.M. Rates of nucleotide substitution vary greatly among plant mitochondrial, chloroplast, and nuclear DNAs. Proc. Natl. Acad. Sci. USA 1987, 84, 9054-9058. [CrossRef] [PubMed] 
13. Drouin, G.; Daoud, H.; Xia, J. Relative rates of synonymous substitutions in the mitochondrial, chloroplast and nuclear genomes of seed plants. Mol. Phylogenetics Evol. 2008, 49, 827-831. [CrossRef] [PubMed]

14. Smith, D.R. Mutation Rates in Plastid Genomes: They Are Lower than You Might Think. Genome Biol. Evol. 2015, 7, 1227-1234. [CrossRef] [PubMed]

15. Chumley, T.W.; Palmer, J.D.; Mower, J.P.; Fourcade, H.M.; Calie, P.J.; Boore, J.L.; Jansen, R.K. The complete chloroplast genome sequence of Pelargonium $x$ hortorum: Organization and evolution of the largest and most highly rearranged chloroplast genome of land plants. Mol. Biol. Evol. 2006, 23, 2175-2190. [CrossRef] [PubMed]

16. Zhou, Y.; Nie, J.; Xiao, L.; Hu, Z.; Wang, B. Comparative Chloroplast Genome Analysis of Rhubarb Botanical Origins and the Development of Specific Identification Markers. Molecules 2018, 23, 2811. [CrossRef] [PubMed]

17. Park, I.; Yang, S.; Kim, W.J.; Noh, P.; Lee, H.O.; Moon, B.C. The Complete Chloroplast Genomes of Six Ipomoea Species and Indel Marker Development for the Discrimination of Authentic Pharbitidis Semen (Seeds of I. nil or I. purpurea). Front. Plant Sci. 2018, 9, 965. [CrossRef] [PubMed]

18. Chen, X.; Cui, Y.; Nie, L.; Hu, H.; Xu, Z.; Sun, W.; Gao, T.; Song, J.; Yao, H. Identification and Phylogenetic Analysis of the Complete Chloroplast Genomes of Three Ephedra Herbs Containing Ephedrine. Biomed. Res. Int. 2019, 2019, 5921725. [CrossRef] [PubMed]

19. Lemieux, C.; Otis, C.; Turmel, M. Ancestral chloroplast genome in Mesostigma viride reveals an early branch of green plant evolution. Nature 2000, 403, 649-652. [CrossRef] [PubMed]

20. Zhu, S.; Niu, Z.; Xue, Q.; Wang, H.; Xie, X.; Ding, X. Accurate authentication of Dendrobium officinale and its closely related species by comparative analysis of complete plastomes. Acta Pharm. Sin. B 2018, 8, 969-980. [CrossRef] [PubMed]

21. Wu, M.L.; Li, Q.; Xu, J.; Li, X.W. Complete chloroplast genome of the medicinal plant Amomum compactum: gene organization, comparative analysis, and phylogenetic relationships within Zingiberales. Chin. Med. 2018, 13, 1875. [CrossRef] [PubMed]

22. Moore, M.J.; Soltis, P.S.; Bell, C.D.; J Gordon, B.; Soltis, D.E. Phylogenetic analysis of 83 plastid genes further resolves the early diversification of eudicots. PNAS 2010, 107, 4623-4628. [CrossRef] [PubMed]

23. Xiang, B.; Li, X.; Qian, J.; Wang, L.; Ma, L.; Tian, X.; Wang, Y. The Complete Chloroplast Genome Sequence of the Medicinal Plant Swertia mussotii Using the PacBio RS II Platform. Molecules 2016, 21, 1029. [CrossRef] [PubMed]

24. Wu, M.; Li, Q.; Hu, Z.; Li, X.; Chen, S. The Complete Amomum kravanh Chloroplast Genome Sequence and Phylogenetic Analysis of the Commelinids. Molecules 2017, 22, 1875. [CrossRef] [PubMed]

25. Jiang, D.; Zhao, Z.; Zhang, T.; Zhong, W.; Liu, C.; Yuan, Q.; Huang, L. The Chloroplast Genome Sequence of Scutellaria baicalensis Provides Insight into Intraspecific and Interspecific Chloroplast Genome Diversity in Scutellaria. Genes (Basel) 2017, 8, 227. [CrossRef]

26. Zuo, L.H.; Shang, A.Q.; Zhang, S.; Yu, X.Y.; Ren, Y.C.; Yang, M.S.; Wang, J.M. The first complete chloroplast genome sequences of Ulmus species by de novo sequencing: Genome comparative and taxonomic position analysis. PLoS ONE 2017, 12, e0171264. [CrossRef] [PubMed]

27. Zhou, J.; Cui, Y.; Chen, X.; Li, Y.; Xu, Z.; Duan, B.; Li, Y.; Song, J.; Yao, H. Complete Chloroplast Genomes of Papaver rhoeas and Papaver orientale: Molecular Structures, Comparative Analysis and Phylogenetic Analysis. Molecules 2018, 23, 437. [CrossRef] [PubMed]

28. Yang, Z.; Huang, Y.; An, W.; Zheng, X.; Huang, S.; Liang, L. Sequencing and Structural Analysis of the Complete Chloroplast Genome of the Medicinal Plant Lycium chinense Mill. Plants (Basel) 2019, 8, 87. [CrossRef] [PubMed]

29. Powell, W.; Morgante, M.; Mcdevitt, R.; Vendramin, G.G.; Rafalski, J.A. Polymorphic simple sequence repeat regions in chloroplast genomes: Applications to the population genetics of pines. PNAS 1995, 92, 7759-7763. [CrossRef] [PubMed]

30. Yang, A.H.; Zhang, J.J.; Yao, X.H.; Huang, H.W. Chloroplast microsatellite markers in Liriodendron tulipifera (Magnoliaceae) and cross-species amplification in L. chinense. Am. J. Bot. 2011, 98, 123-126. [CrossRef] [PubMed]

31. Jiao, Y.J.H.; Li, X.W.; Chai, M.L.; Jia, H.J.; Chen, Z.; Wang, G.Y.; Chai, C.Y.; van de Weg, E.; Gao, Z.S. Development of simple sequence repeat (SSR) markers from a genome survey of Chinese bayberry (Myrica rubra). BMC Genom. 2012, 13, 201. [CrossRef] 
32. Jianhua, X.; Shuo, W.; Shi-Liang, Z. Polymorphic chloroplast microsatellite loci in Nelumbo (Nelumbonaceae). Am. J. Bot. 2012, 99, 240-244. [CrossRef]

33. Park, I.; Yang, S.; Choi, G.; Kim, W.J.; Moon, B.C. The Complete Chloroplast Genome Sequences of Aconitum pseudolaeve and Aconitum longecassidatum, and Development of Molecular Markers for Distinguishing Species in the Aconitum Subgenus Lycoctonum. Molecules 2017, 22, 2012. [CrossRef] [PubMed]

34. Tonti-Filippini, J.; Nevill, P.G.; Dixon, K.; Small, I. What can we do with 1000 plastid genomes? Plant J. 2017, 90, 808-818. [CrossRef] [PubMed]

35. NCBI. Genome. Available online: https://www.ncbi.nlm.nih.gov/genome/?term= (accessed on 26 June 2019).

36. Bolger, A.M.; Lohse, M.; Usadel, B. Trimmomatic: A flexible trimmer for Illumina sequence data. Bioinformatics 2014, 30, 2114-2120. [CrossRef] [PubMed]

37. Jackman, S.D.; Vandervalk, B.P.; Mohamadi, H.; Chu, J.; Yeo, S.; Hammond, S.A.; Jahesh, G.; Khan, H.; Coombe, L.; Warren, R.L. ABySS 2.0: Resource-efficient assembly of large genomes using a Bloom filter. Genome Res. 2017, 27, 768-777. [CrossRef] [PubMed]

38. Luo, R.; Liu, B.; Xie, Y.; Li, Z.; Huang, W.; Yuan, J.; He, G.; Chen, Y.; Pan, Q.; Liu, Y. SOAPdenovo2: An empirically improved memory-efficient short-read de novo assembler. Gigascience 2012, 1, 18. [CrossRef] [PubMed]

39. Boetzer, M.; Henkel, C.V.; Jansen, H.J.; Butler, D.; Pirovano, W. Scaffolding pre-assembled contigs using SSPACE. Bioinformatics 2011, 27, 578-579. [CrossRef] [PubMed]

40. Xu, G.C.; Xu, T.J.; Zhu, R.; Zhang, Y.; Li, S.Q.; Wang, H.W.; Li, J.T. LR_Gapcloser: A tiling path-based gap closer that uses long reads to complete genome assembly. Gigascience 2019, 8. [CrossRef]

41. Liu, C.; Shi, L.; Zhu, Y.; Chen, H.; Zhang, J.; Lin, X.; Guan, X. CpGAVAS, an integrated web server for the annotation, visualization, analysis, and GenBank submission of completely sequenced chloroplast genome sequences. BMC Genomics 2012, 13, 715. [CrossRef]

42. Wyman, S.K.; Jansen, R.K.; Boore, J.L. Automatic annotation of organellar genomes with DOGMA. Bioinformatics 2004, 20, 3252-3255. [CrossRef]

43. Peter, S.; Brooks, A.N.; Lowe, T.M. The tRNAscan-SE, snoscan and snoGPS web servers for the detection of tRNAs and snoRNAs. Nucleic Acids Res. 2005, 33, 686-689. [CrossRef]

44. Lohse, M.; Drechsel, O.; Bock, R. OrganellarGenomeDRAW (OGDRAW): A tool for the easy generation of high-quality custom graphical maps of plastid and mitochondrial genomes. Curr. Genet. 2007, 52, 267-274. [CrossRef] [PubMed]

45. Tamura, K.; Stecher, G.; Peterson, D.; Filipski, A.; Kumar, S. MEGA6: Molecular Evolutionary Genetics Analysis version 6.0. Mol. Biol. Evol. 2013, 30, 2725-2729. [CrossRef] [PubMed]

46. Beier, S.; Thiel, T.; Münch, T.; Scholz, U.; Mascher, M. MISA-web: A web server for microsatellite prediction. Bioinformatics 2017, 33, 2583-2585. [CrossRef] [PubMed]

47. Kurtz, S.; Schleiermacher, C. REPuter: Fast computation of maximal repeats in complete genomes. Bioinformatics 1999, 15, 426-427. [CrossRef] [PubMed]

48. Katoh, K.; Rozewicki, J.; Yamada, K.D. MAFFT online service: Multiple sequence alignment, interactive sequence choice and visualization. Brief. Bioinform. 2017. [CrossRef] [PubMed]

49. Frazer, K.A.; Lior, P.; Alexander, P.; Rubin, E.M.; Inna, D. VISTA: Computational tools for comparative genomics. Nucleic Acids Res. 2004, 32. [CrossRef]

50. Rozas, J.; Ferrermata, A.; Sánchezdelbarrio, J.C.; Guiraorico, S.; Librado, P.; Ramosonsins, S.E.; Sánchezgracia, A. Evolution. DnaSP 6: DNA Sequence Polymorphism Analysis of Large Datasets. Mol. Biol. 2017, 34, 3299-3302. [CrossRef]

51. Nguyen, L.T.; Schmidt, H.A.; Von Haeseler, A.; Minh, B.Q. IQ-TREE: A Fast and Effective Stochastic Algorithm for Estimating Maximum-Likelihood Phylogenies. Mol. Biol. Evol. 2015, 32, 268-274. [CrossRef]

(C) 2019 by the authors. Licensee MDPI, Basel, Switzerland. This article is an open access article distributed under the terms and conditions of the Creative Commons Attribution (CC BY) license (http://creativecommons.org/licenses/by/4.0/). 